\title{
REVIEW
}

\section{Fracture healing and lipid mediators}

\section{J Patrick O'Connor, Michaele B Manigrasso', Brian D Kim and Sangeeta Subramanian}

\author{
Department of Biochemistry \& Molecular Biology, New Jersey Medical School and Graduate School of Biomedical \\ Sciences, Rutgers, the State University of New Jersey, Newark, NJ, USA.
}

Lipid mediators regulate bone regeneration during fracture healing. Prostaglandins and leukotrienes are well-known lipid mediators that regulate inflammation and are synthesized from the $\Omega-6$ fatty acid, arachidonic acid.

Cyclooxygenase (COX-1 or COX-2) and 5-lipoxygenase (5-LO) catalyze the initial enzymatic steps in the synthesis of prostaglandins and leukotrienes, respectively. Inhibition or genetic ablation of COX-2 activity impairs fracture healing in animal models. Genetic ablation of COX-1 does not affect the fracture callus strength in mice, suggesting that COX-2 activity is primarily responsible for regulating fracture healing. Inhibition of cyclooxygenase activity with nonsteroidal anti-inflammatory drugs (NSAIDs) is performed clinically to reduce heterotopic ossification, although clinical evidence that NSAID treatment impairs fracture healing remains controversial. In contrast, inhibition or genetic ablation of 5-LO activity accelerates fracture healing in animal models. Even though prostaglandins and leukotrienes regulate inflammation, loss of COX-2 or 5-LO activity appears to primarily affect chondrogenesis during fracture healing. Prostaglandin or prostaglandin analog treatment, prostaglandin-specific synthase inhibition and prostaglandin or leukotriene receptor antagonism also affect callus chondrogenesis. Unlike the $\Omega$-6-derived lipid mediators, lipid mediators derived from $\Omega-3$ fatty acids, such as resolvin $\mathrm{E}_{1}$ (RvE1), have anti-inflammatory activity. In vivo, RvE1 can inhibit osteoclastogenesis and limit bone resorption. Although $\Omega-6$ and $\Omega-3$ lipid mediators have clear-cut effects on inflammation, the role of these lipid mediators in bone regeneration is more complex, with apparent effects on callus chondrogenesis and bone remodeling.

BoneKEy Reports 3, Article number: 517 (2014) | doi:10.1038/bonekey.2014.12

\section{Introduction}

Bone fracture healing recapitulates many events associated with fetal endochondral bone development. However, inflammation is an early physiological response to a fracture that does not appear to occur during fetal bone development or during normal adolescent bone growth. ${ }^{1}$ This suggests that events associated with inflammation may be necessary for fracture healing to occur successfully. Indeed, nonsteroidal anti-inflammatory drugs (NSAIDs) that inhibit inflammation also impair fracture healing. ${ }^{2}$ NSAIDs reduce the synthesis of lipid mediators that regulate inflammation by inhibiting cyclooxygenase activity. This indicates that cyclooxygenase-derived lipid mediators have a critical role in promoting fracture healing. Understanding how lipid mediators govern fracture healing is necessary to identify novel approaches to promote bone regeneration and better patient care.

\section{Lipid Mediator Synthesis from AA, EPA and DHA}

Lipid mediators are bioactive lipids involved in cell signaling and are known to regulate a variety of physiological and pathological processes. ${ }^{3}$ Those lipid mediators derived from $\Omega-6$ fatty acids that include arachidonic acid (AA; 20:4n-6) and $\Omega-3$ fatty acids that include eicosapentaenoic acid (EPA; 22:5n-3) and docosahexaenoic acid (DHA; 22:6n-3) are known to be potent regulators of inflammation. ${ }^{4}$ In general, $\Omega$-6-derived lipid mediators promote inflammation, whereas $\Omega$-3-derived lipid mediators resolve inflammation. As described below, manipulation of these pathways can profoundly impair or promote bone regeneration.

$A A, E P A$ and DHA are stored in cell membranes as phospholipids. Thus, the initial step in utilizing these substrates for lipid mediator synthesis is to hydrolyze AA, EPA or DHA from phospholipids. The hydrolysis reaction can be catalyzed

Correspondence: Professor JP O'Connor, Department of Biochemistry \& Molecular Biology, New Jersey Medical School and Graduate School of Biomedical Sciences, Rutgers, the State University of New Jersey, Medical Sciences Building room E-659, 185 South Orange Avenue, Newark, NJ 07103, USA

E-mail: oconnojp@rutgers.edu

${ }^{1}$ Present address: New York University Langone Medical Center, New York, NY, USA 
by several phospholipases. $^{5}$ However, the Group IVA calcium-dependent cytosolic phospholipase (Pla2g4a) appears to preferentially hydrolyze $A A$ and can also hydrolyze EPA from phospholipids. ${ }^{6}$ In contrast, hydrolysis of DHA from phospholipids appears to be preferentially performed by phospholipases other than cytosolic phospholipase, such as secreted phospholipase $A_{2}$ (Pla2g2a). ${ }^{6}$

Once hydrolyzed, AA, DHA and EPA are either rapidly reinserted into the lipid membrane by lysophospholipid acyltransferases via the Lands cycle or are converted into lipid mediator intermediates by cyclooxygenase, lipoxygenase or cytochrome P450 catalysis as summarized in Figure 1. The intermediates are converted into the active lipid mediators by a variety of enzymatic and nonenzymatic steps that can include transcellular reactions. For instance, the intermediate leukotriene $\mathrm{A}_{4}\left(\mathrm{LTA}_{4}\right)$ can be secreted by neutrophils and converted into active leukotriene $\mathrm{B}_{4}\left(\mathrm{LTB}_{4}\right)$ by $\mathrm{LTA}_{4}$ hydrolase expressed in chondrocytes. ${ }^{7}$ Loss of a specific synthetic enzyme has sometimes been associated with elevated synthesis of other lipid mediators through presumed intermediate shunting mechanisms. $^{8-10}$ Thus, one cannot assume that by inhibiting the activity of one enzyme only the immediate and downstream products of that enzyme will be affected. Indeed, nontargeted

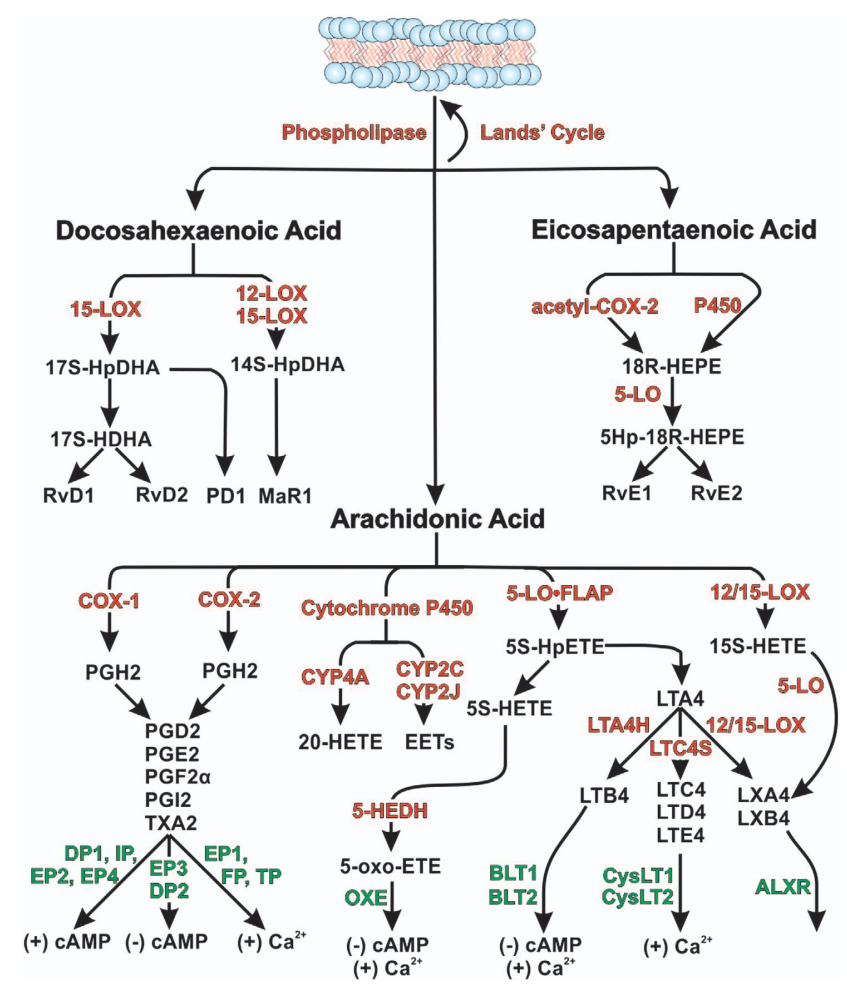

Figure 1 Pathways for synthesis and signaling of arachidonic acid, docosahexaenoic acid and eicosapentaenoic acid-derived lipid mediators. The diagram summarizes some of the synthetic pathways for lipid mediator synthesis. Enzyme activities are shown in red text and include cyclooxygenase (COX), 5-lipoxygenase (5-LO or 5-LO·FLAP), lipoxygenase, cytochrome P450, LTA4 hydrolase, LTC4 synthase, 5-hydroxy-eicosanoid dehydrogenase and COX-2 covalently modified by acetylsalicylic acid. Receptors are shown in green text. Lipid mediators and synthetic intermediates are shown in black text and include resolvins, protectin D1, maresin-1, prostaglandins, leukotrienes, lipoxins, 5-oxo-eicosatetraenoic acid, 20-hydroxyeicosatetraenoic acid, various epoxyeicosatrienoic acids and others. Known effects of receptor activation on intracellular cyclic adenosine monophosphate or calcium levels are shown at the bottom lipid mediator levels also should be measured to assess any shunting effects. After synthesis, the lipid mediators are secreted and signal or they are rapidly converted into excreted metabolites. Activation of AA-derived lipid mediator receptors typically regulates intracellular cyclic adenosine monophosphate (CAMP) production or intracellular calcium levels depending upon whether the receptor preferentially interacts

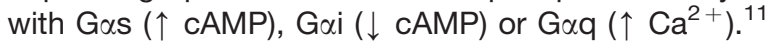

\section{The Role of Lipid Mediator Synthesis Enzymes in Fracture Healing}

\section{Cyclooxygenase-2}

Two cyclooxygenase isoforms, COX-1 and COX-2, catalyze critical steps in the synthesis of pro-inflammatory prostaglandins from AA. Inhibition of COX-2 has a clear detrimental effect on bone fracture healing in animal models. In several studies, fracture healing was delayed in animals treated with NSAIDs in that intermediate measures of healing were reduced in the NSAID-treated animals but ultimate healing was not significantly affected. ${ }^{2}$ In contrast, fracture healing was severely impaired in rats treated with COX-2-selective NSAIDs, such as rofecoxib and celecoxib. ${ }^{12}$ Healing in the COX-2-selective NSAID-treated rats was characterized by abnormal callus cartilage morphology and an apparent failure of the callus chondrocytes to progress into hypertrophy based upon reduced type $X$ collagen $m R N A$ levels. ${ }^{13}$ Similar effects on type $X$ collagen mRNA levels also have been observed in COX-2-null mice. ${ }^{14}$ It was hypothesized that the failed chondrocyte hypertrophy stops endochondral bone formation, which leads to fracture nonunion. ${ }^{13}$

In humans, NSAIDs, including COX-2-selective NSAIDs, are used therapeutically to limit heterotopic bone formation following hip fracture repairs. The heterotopic bone develops, at least in part, through an endochondral ossification pathway. In this regard, NSAIDs can clearly inhibit bone formation in humans. However, data regarding the effects of NSAIDs on fracture healing are less compelling as recently reviewed by Kurmis et al. ${ }^{15}$ Burd et al. ${ }^{16}$ found that 6 weeks of indomethacin therapy to reduce heterotopic ossification following hip fracture caused a significant increase in the development of fracture nonunions in different bones as compared with patients who received localized radiation to control heterotopic ossification (29\% vs $7 \%$ ). In most fracture patients, however, 6 weeks of indomethacin or other NSAID therapy to control pain may not be necessary. Further, it is unclear whether those patients who experience delayed fracture unions do so because of an extended period of NSAID use or whether the extended NSAID use is to control the symptoms of the delayed union brought about by other causes, and perhaps exacerbated by NSAID use. As NSAID dose and duration of treatment are critical factors in the development of fracture nonunions in animal models, understanding when NSAID therapy should be avoided during fracture healing could be critical for managing patient treatment. $^{17}$

Attempts to enhance fracture healing by overexpressing COX-2 at the fracture site have been performed. Using a retroviral vector to express human COX-2, rat fracture calluses injected with the COX-2 retrovirus appeared to heal faster. ${ }^{18}$ In contrast, when mesenchymal cells were transduced with a retroviral vector expressing human COX-2 ex vivo and then 
transplanted into a murine calvarial defect, the COX-2expressing cells had no effect on healing but reduced bone mineral density when coadministered with mesenchymal cells transduced to express human bone morphogenetic protein $4 .^{19}$ Pulsed ultrasound also has been shown to increase COX-2 expression at mouse fracture sites, suggesting that extracorporeal mechanical stimulation of fracture healing may be mediated by enhanced COX-2 activity. ${ }^{20}$

\section{Cyclooxygenase-1}

Few studies have specifically examined the effects of lost COX1 activity on fracture healing. Preliminary experiments in COX1-null mice showed that these mice developed normal fracture calluses suggesting that COX-1 is not critical for fracture healing. ${ }^{12,21}$ We performed a more in-depth analysis of fracture healing in COX-1-null mice, which included histomorphometry at 7, 10, 14 and 21 days after fracture (Figure 2) and torsional mechanical testing at 4 weeks (Figure 3) and 12 weeks (not shown). Whereas the COX-2-null calluses were smaller with less cartilage and bone, no differences between COX-1-null and control values were observed. Similarly, the COX-2-null peak torque, maximum rigidity, maximum shear stress and shear modulus values were significantly lower than control values $(P<0.001)$ at 4 weeks after fracture, but no difference was observed between control and COX-1-null values. At 12 weeks after fracture, the COX-2-null maximum rigidity and maximum shear stress values remained significantly lower than control values $(P<0.005)$, but again no difference was observed between control and COX-1-null values. Thus, any negative effect of NSAID treatment on fracture healing likely relates to the inhibition of COX-2 by that NSAID rather than COX-1. Nevertheless, loss of COX-1 activity does affect fracture callus biology as noted below.

\section{Prostaglandin synthases}

Prostaglandin $\mathrm{H}_{2}\left(\mathrm{PGH}_{2}\right)$ is the intermediary prostaglandin produced by cyclooxygenase and is converted into the bioactive lipid mediators $\mathrm{PGD}_{2}, \mathrm{PGE}_{2}, \mathrm{PGF}_{2 \alpha}, \mathrm{PGI}_{2}$ and thromboxane $\mathrm{A}_{2}$ by specific synthases. ${ }^{22}$ Altering the activity of these synthases can affect many inflammation-related processes. In terms of fracture healing, only microsomal prostaglandin $E_{2}$ synthase-1 (mPGES-1) has been specifically studied. Genetic ablation of mPGES-1 in mice has no apparent effects on normal skeletal development but causes a significant impairment in tibial fracture healing. ${ }^{23}$ Tibial fractures in 5 of 10 mPGES-1-null mice failed to heal after 21 days, whereas 10 of 10 fractures healed in control mice. Levels of mPGES-1 mRNA peaked at 7 days after fracture in the mouse tibiae, which correlates with the peak period of callus cartilage formation. Consistent with this expression pattern, callus chondrocyte proliferation was significantly reduced in mPGES-1-null mice. This suggests that mPGES-1 promotes fracture healing through regulation of callus chondrogenesis. Interestingly, prostaglandin $\mathrm{I}_{2}$ synthase-null mice have decreased bone mass as juveniles (5 weeks old) but elevated bone mass and bone formation rates as adults (34 weeks old). ${ }^{24}$ This suggests that prostaglandin $\mathrm{I}_{2}$ synthase may be a therapeutic target for enhancing bone formation.

\section{5-Lipoxygenase (5-LO)}

Inhibition of cyclooxygenase activity can shunt AA into the 5-LO pathway leading to increased leukotriene synthesis. ${ }^{9}$ Indeed,
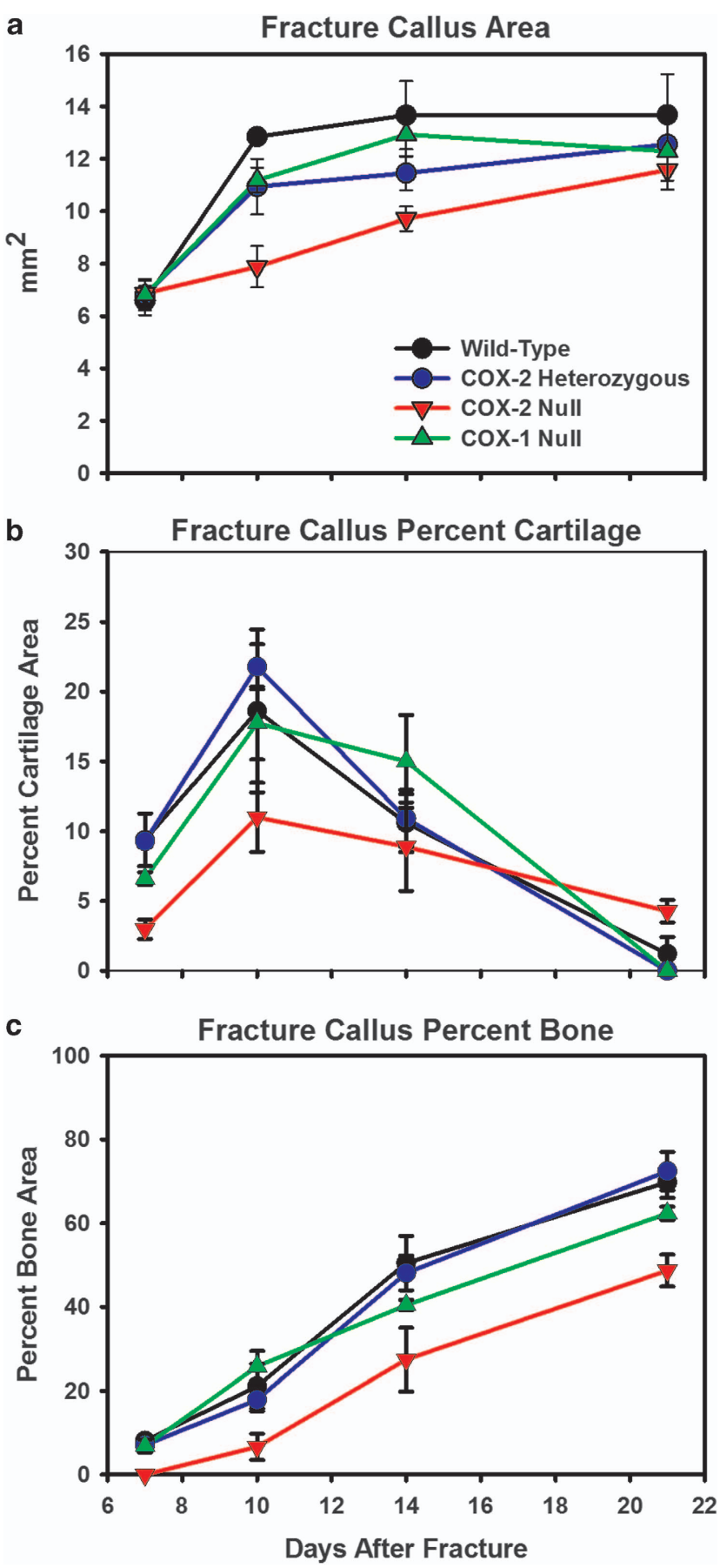

Figure 2 Histomorphometric analysis of fracture healing in COX-deficient mice

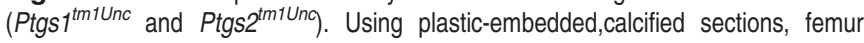
fracture callus area (a), callus percent cartilage area $(\mathbf{b})$ and callus percent mineralized tissue area (c) were measured and calculated at 7, 10, 14 and 21 days after fracture. Shown are mean values and standard errors ( $n \geq 3$ for all time points and genotypes). Callus area, percent cartilage area and percent mineralized tissue area were reduced in COX-2-null mice as expected. However, no significant differences were detected between COX-1-null, wild-type or mice heterozygous for the COX-2-null allele.

fracture calluses of COX-2-null mice have significantly higher levels of $\mathrm{LTB}_{4}$. Addition of $\mathrm{LTB}_{4}$ or especially 5-hydroxyeicosatetraenoic acid to cultured calvarial cells impairs bone formation in vitro. ${ }^{25,26}$ Further, bone cortical thickness is increased in mice lacking 5-LO, again suggesting that 5-LO lipid 
mediators impair bone formation. ${ }^{27}$ Thus, it was hypothesized that the impaired fracture healing response observed in NSAIDtreated animals or in COX-2-null mice may be caused by excessive 5-LO lipid mediator synthesis. This hypothesis was
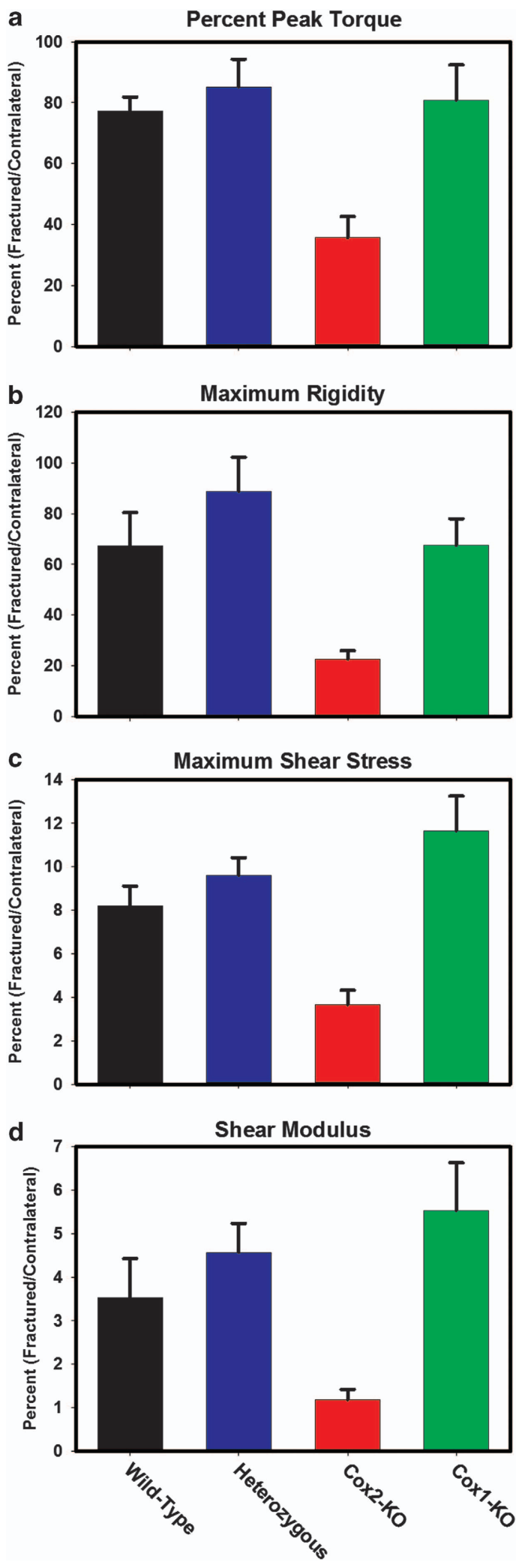

tested by measuring the fracture healing response in rats treated with AA-861, a 5-LO inhibitor, and in 5-LO-null mice. 9,13 In both cases, fracture healing was significantly accelerated with an $\sim 25 \%$ reduction in fracture callus bridging time relative to placebo-treated rats or control mice and with concomitant increases in callus mechanical properties. Cartilage formation and progression into hypertrophy appeared to be enhanced in the 5-LO-deficient animals, suggesting that endochondral ossification was occurring earlier and faster to heal the fracture. Although the experimental results support the hypothesis, the mechanism by which inhibition of 5-LO accelerates healing is not known. Suppression of 5-LO lipid mediator synthesis could contribute to promoting fracture healing, but inhibition of 5-LO also caused a significant increase in fracture callus COX-2 mRNA levels that could aid in healing.

5-LO-activating protein (FLAP) is an essential cofactor for 5LO activity. Loss or inhibition of FLAP does inhibit 5-LO activity but the effects of FLAP inhibition on bone biology have not been investigated.

Once 5-LO has synthesized LTA 4 , the LTA 4 is converted into $\mathrm{LTB}_{4}$ by LTA 4 hydrolase or into leukotriene $\mathrm{C}_{4}\left(\mathrm{LTC}_{4}\right)$ by $\mathrm{LTC}_{4}$ synthase. $\mathrm{LTC}_{4}$ is subsequently modified to create leukotriene $D_{4}$ and leukotriene $E_{4}$. The effects of inhibiting these enzyme activities on fracture healing have not been reported. However, $\mathrm{LTA}_{4}$ hydrolase and $\mathrm{LTC}_{4}$ synthase are expressed in chondrocytes and can mediate transcellular synthesis of $\mathrm{LTB}_{4}$ and $\mathrm{LTC}_{4}{ }^{7}$ Interestingly, LTA 4 hydrolase shares many structural properties with angiotensin-converting enzyme (ACE) such that some ACE inhibitors can also inhibit LTA 4 hydrolase and certain ACE inhibitors have been shown to promote fracture healing. ${ }^{28}$

\section{Other lipid mediator synthesis enzymes}

Variations in human and mouse bone mass have been associated with 12- and 15-lipoxygenase activity. In mice, a single gene, Alox15, encodes a protein with both 12- and 15-lipoxygenase activity, and genetic or pharmacological inhibition of 12/15-LO activity increased bone mineral density in mice. ${ }^{29}$ In separate studies, variations in human bone mineral density have been associated with polymorphisms in the 12-lipoxygenase and 15-lipoxygenase genes. ${ }^{30,31}$ However, no published reports describe how loss of 12/15-LO activity affects fracture healing. In addition, although many other enzymes involved in synthesis of AA-, EPA- and DHA-derived lipid mediators have been investigated for their roles in inflammation and inflammation-related pathologies, no published reports of how these enzymes affect fracture healing are available.

\section{Mechanistic interpretation}

The mechanism by which COX-2 and associated lipid mediators regulate fracture healing remains unknown. As indicated

Figure 3 Mechanical testing analysis of fractured femurs from COX-deficient mice. Fractured femurs and contralateral control femurs were collected from COX-1-null, COX-2-null, wild-type and mice heterozygous for the COX-2-null allele after 4 weeks of healing. The femurs were analyzed for structural (peak torque and maximum rigidity, $\mathbf{a}$ and $\mathbf{b}$ ) and material properties (maximum shear stress and shear modulus, $\mathbf{c}$ and $\mathbf{d}$ ) by torsional mechanical testing to failure. Each fractured femur value was normalized to its contralateral femur value as a percentage and the means with standard errors for each genotype are shown $(n \geq 9)$. For the COX-2-deficient mice, all values were significantly less than the values from other genotypes $(P<0.05)$. However, no significant differences were detected between the COX-1-null mice and controls. 
above, COX-2 inhibition appears to affect callus chondrocyte function, leading to impaired endochondral ossification. ${ }^{13}$ However, other studies have suggested that COX-2 is critical for differentiation of mesenchymal cells into osteoblasts. ${ }^{21,32} \mathrm{COX}$ 2 mRNA levels at the fracture site increase immediately after fracture but peak during the regenerative, rather than inflammatory, phase of healing. ${ }^{13,14,33}$ Few studies have actually measured lipid mediator levels during fracture healing. Dekel et al. ${ }^{34}$ measured prostaglandin $\mathrm{E}$ and $\mathrm{F}$ levels from the bone and surrounding muscle of rabbit tibial fractures. Prostaglandin $E$ levels released from the bone increased almost fourfold on day 3 after fracture as compared with controls, whereas prostaglandin $\mathrm{F}$ levels increased about threefold from day 3 to day 7 in the fractured bone. Significantly higher prostaglandin $\mathrm{E}$ and $\mathrm{F}$ levels were detected in the surrounding muscle, with $E$ levels peaking at day 7 and $F$ levels peaking at day 14 , relative to controls. In mice with targeted mutations of the COX-1, COX-2 or 5-LO genes, significant changes in callus prostaglandin $E_{2}$ and $F_{2 \alpha}$ and $\mathrm{LTB}_{4}$ levels were found. ${ }^{9}$ Unexpectedly, loss of the COX-1 or COX-2 enzyme led to elevated mouse femur fracture callus $\mathrm{PGE}_{2}$ levels at day 4 after fracture. In contrast, $\mathrm{PGF}_{2 \alpha}$ levels were near zero in the COX-2null calluses, whereas $\mathrm{LTB}_{4}$ levels were markedly elevated. In rat fracture calluses, $\mathrm{PGE}_{2}$ levels peaked at day 1 after fracture before declining to baseline levels by day $14 .{ }^{35}$ Interestingly, in rats treated with keterolac or valdecoxib for 7 days after fracture, $\mathrm{PGE}_{2}$ levels were greater than those of placebo controls at 14 days after fracture, indicating that the normal pattern of COX-2 expression is disrupted by NSAID therapy. ${ }^{35}$ In humans, increased levels of urine $11 \beta-\mathrm{PGF}_{2 \alpha}$, a metabolite of $\mathrm{PGD}_{2}$, were detected in fracture patients 5-6 weeks after fracture. ${ }^{22}$ Together these observations indicate that pharmacological or genetic inhibition of the enzymes responsible for lipid mediator synthesis alters the temporal or cellular pattern of expression and activity of COX-1, COX-2 and 5-LO. As the normal pattern of COX-1, COX-2 and 5-LO expression and activity has yet to be clearly defined, the consequences of disrupting the activity of one or more of these enzymes on lipid mediator synthesis remains to be determined and to be correlated to fracture-healing outcomes.

\section{The Role of Lipid Mediator Receptors in Fracture Healing}

The AA-derived lipid mediators signal through G-proteincoupled receptors to affect intracellular cAMP and $\mathrm{Ca}^{2}+$ levels. As shown in Figure 1, receptors for several of the AA-derived lipid mediators have been identified. As modulating the activity of AA-derived lipid mediator synthesis enzymes can alter fracture healing, similar effects would be expected by modulating the activity of the receptors for those lipid mediators. However, only a few published studies have been performed to test this contention.

Mouse models with targeted null mutations in the prostaglandin and leukotriene receptors have been developed. ${ }^{36,37}$ Published reports specifically testing whether loss of a lipid mediator receptor would affect fracture healing have focused on the $\mathrm{PGE}_{2}$ receptors. Activation of the $\mathrm{PGE}_{2}$ receptor prostaglandin $\mathrm{E}$ receptor 1 (EP1) increases intracellular $\mathrm{Ca}^{2+}$; activation of EP2 or EP4 increases cAMP levels; and activation of EP3 decreases cAMP levels. Aged EP4-null mice (15-16 months old) have significantly reduced bone mass and show impaired fracture healing characterized by small callus formation with reduced amounts of cartilage that persists longer than in age-matched controls. ${ }^{38}$ Conversely, fracture healing appears to be accelerated in mice null for EP1. ${ }^{39}$ In this case, loss of EP1 function is characterized by an early abundance of callus cartilage formation that more rapidly progresses into hypertrophy, similar to what was observed in 5-LO-null mice. ${ }^{9}$

The effects of prostaglandin receptor agonists on fracture healing generally support the fracture healing phenotypes of the receptor-null mice. An EP2 agonist, CP-533536, was found to enhance rat femur fracture healing and to promote canine segmental defect and osteotomy healing. ${ }^{40,41}$ An EP4 agonist, ONO-4819, was also shown to promote healing of rat femur cortical defects and enhance the activity of bone morphogenetic protein 2 during rabbit spinal fusion. ${ }^{42,43}$ When fractures in COX-2-null mice were treated with an EP2 (CP-463755) or an EP4 (CP-734432) agonist, the EP4 agonist rescued fracture healing in the COX-2-null mice better than did the EP2 agonist. ${ }^{14}$

The effects of montelukast treatment on fracture healing have also been studied. Montelukast antagonizes cysteinyl leukotriene receptor 1 and is commonly used to treat asthma. ${ }^{44,45}$ Mice treated with montelukast daily $\left(1.5 \mathrm{mg} \mathrm{kg}^{-1}\right.$, gastro-intestinal gavage) showed increased fracture callus cartilage amounts similar to what had been observed in 5-LOnull mice or in rats treated with a 5-LO inhibitor. ${ }^{9,13,46}$ In contrast, when rats were treated with montelukast daily $\left(1 \mathrm{mg} \mathrm{kg}^{-1}\right.$, intraperitoneal injection), no effect on tibial fracture healing was observed. ${ }^{47}$ The doses of montelukast used were several-fold higher than those used in humans $\left(\sim 0.15 \mathrm{mg} \mathrm{kg}^{-1}\right.$ per day). This is relevant in that high concentrations of montelukast can also inhibit 5-LO activity in addition to antagonizing cysteinyl leukotriene receptor $1 .^{48}$ Thus, it is unclear whether the positive effects of montelukast on fracture healing are specific only to mice and whether the effect can be attributed to cysteinyl leukotriene receptor 1 antagonism or 5-LO inhibition.

Many AA-derived lipid mediator receptor agonists and antagonists have been evaluated for effects on osteoblasts, chondrocytes, endothelial cells and osteoclasts in vitro. Fewer have been tested for in vivo effects, although bone formation was identified as a side effect of using $\mathrm{PGE}_{1}$ to treat ductus arteriosus in children. ${ }^{49}$ Still, many of the lipid mediator agonists and antagonists have promising, potential therapeutic effects. For instance, RvE1 can impair osteoclastogenesis and, unlike $\mathrm{PGE}_{2}$ or $\mathrm{LTB}_{4}$, local application of RvE1 can limit bone destruction associated with periodontal disease. ${ }^{50}$ However, direct testing of RvE1 and many other agonists and antagonists on fracture healing remains to be performed.

\section{Conclusions}

As described above, numerous animal studies support a role for $\Omega-6$ and $\Omega-3$ fatty acid-derived lipid mediators in bone biology and fracture healing. To a great extent, the cited material was limited to in vivo studies. However, many reports describe the effects of lipid mediators on osteoclasts, osteoblasts and chondrocytes and in some circumstances support and, in others, contradict the in vivo studies. The contradictions may, in part, reflect crucial gaps in our knowledge of lipid mediators that regulate bone homeostasis and regeneration. Clear 
descriptions of which cells express which lipid mediator synthetic enzymes or receptor during fracture healing are lacking. Similarly, when and to what level each lipid mediator is present during fracture healing remains unknown. Application of current lipidomic analysis methods is likely to provide new insights into how lipid mediators regulate bone homeostasis and regeneration and thereby provide new therapeutic directions.

\section{Conflict of Interest}

JPOC is an owner, board member and officer of Accelalox Inc., which is developing the use of 5-lipoxygenase inhibitors to accelerate fracture healing and promote bone formation. All other authors declare no conflict of interest.

\section{Acknowledgements}

Preparation of this manuscript was supported by Award Number R01DE019926 from the National Institute of Dental \& Craniofacial Research. The content is solely the responsibility of the authors and does not necessarily represent the official views of the National Institute of Dental \& Craniofacial Research or the National Institutes of Health.

\section{References}

1. Schindeler A, McDonald MM, Bokko $P$, Little DG. Bone remodeling during fracture repair: The cellular picture. Semin Cell Dev Biol 2008;19:459-466.

2. Cottrell J, O'Connor JP. Effect of non-steroidal anti-inflammatory drugs on bone healing. Pharmaceuticals 2010;3:1668-1693.

3. Shimizu T. Lipid mediators in health and disease: enzymes and receptors as therapeutic targets for the regulation of immunity and inflammation. Annu Rev Pharmacol Toxicol 2009;49:123-150.

4. Stables MJ, Gilroy DW. Old and new generation lipid mediators in acute inflammation and resolution. Prog Lipid Res 2011;50:35-51.

5. Dennis EA, Cao J, Hsu YH, Magrioti V, Kokotos G. Phospholipase A2 enzymes: physical structure, biological function, disease implication, chemical inhibition, and therapeutic intervention. Chem Rev 2011;111:6130-6185.

6. Shikano M, Masuzawa Y, Yazawa K, Takayama K, Kudo I, Inoue K. Complete discrimination of docosahexaenoate from arachidonate by $85 \mathrm{kDa}$ cytosolic phospholipase A2 during the hydrolysis of diacyl- and alkenylacylglycerophosphoethanolamine. Biochim Biophys Acta 1994:1212:211-216

7. Amat M, Diaz C, Vila L. Leukotriene A4 hydrolase and leukotriene C4 synthase activities in human chondrocytes: transcellular biosynthesis of Leukotrienes during granulocyte-chondrocyte interaction. Arthritis Rheum 1998:41:1645-1651.

8. Trebino CE, Eskra JD, Wachtmann TS, Perez JR, Carty TJ, Audoly LP. Redirection of eicosanoid metabolism in mPGES-1-deficient macrophages. J Biol Chem 2005;280: $16579-16585$.

9. Manigrasso MB, O'Connor JP. Accelerated fracture healing in mice lacking the 5-lipoxygenase gene. Acta Orthop 2010;81:748-755.

10. Duffield-Lillico AJ, Boyle JO, Zhou XK, Ghosh A, Butala GS, Subbaramaiah K et al. Levels of prostaglandin $\mathrm{E}$ metabolite and leukotriene $\mathrm{E}(4)$ are increased in the urine of smokers: evidence that celecoxib shunts arachidonic acid into the 5-lipoxygenase pathway. Cancer Prev Res (Phila Pa) 2009;2:322-329.

11. Hirata T, Narumiya S. Prostanoid receptors. Chem Rev 2011;111:6209-6230.

12. Simon AM, Manigrasso MB, O'Connor JP. Cyclo-oxygenase 2 function is essential for bone fracture healing. J Bone Miner Res 2002;17:963-976.

13. Cottrell JA, O'Connor JP. Pharmacological inhibition of 5-lipoxygenase accelerates and enhances fracture-healing. J Bone Joint Surg Am 2009;91:2653-2665.

14. Xie C, Liang B, Xue M, Lin AS, Loiselle A, Schwarz EM et al. Rescue of impaired fracture healing in COX-2-/- mice via activation of prostaglandin E2 receptor subtype 4. Am J Pathol 2009;175:772-785.

15. Kurmis AP, Kurmis TP, O'Brien JX, Dalen T. The effect of nonsteroidal anti-inflammatory drug administration on acute phase fracture-healing: a review. J Bone Joint Surg Am 2012;94: 815-823.

16. Burd TA, Hughes MS, Anglen JO. Heterotopic ossification prophylaxis with indomethacin increases the risk of long-bone nonunion. J Bone Joint Surg Br 2003;85-B:700-705.

17. Simon AM, O'Connor JP. Dose and time-dependent effects of cyclooxygenase-2 inhibition on fracture-healing. J Bone Joint Surg Am 2007;89:500-511.
18. Rundle $\mathrm{CH}$, Strong DD, Chen ST, Linkhart TA, Sheng MH, Wergedal JE et al. Retroviral-based gene therapy with cyclooxygenase-2 promotes the union of bony callus tissues and accelerates fracture healing in the rat. J Gene Med 2008;10:229-241.

19. Lau KH, Kothari V, Das A, Zhang XB, Baylink DJ. Cellular and molecular mechanisms of accelerated fracture healing by COX2 gene therapy: studies in a mouse model of multiple fractures. Bone 2013:53:369-381.

20. Naruse K, Sekiya H, Harada Y, Iwabuchi S, Kozai Y, Kawamata R et al. Prolonged endochondral bone healing in senescence is shortened by low-intensity pulsed ultrasound in a manner dependent on COX-2. Ultrasound Med Biol 2010;36:1098-1108.

21. Zhang X, Schwarz EM, Young DA, Puzas JE, Rosier RN, O'Keefe RJ. Cyclooxygenase-2 regulates mesenchymal cell differentiation into the osteoblast lineage and is critically involved in bone repair. J Clin Invest 2002;109:1405-1415.

22. Smith WL, Urade Y, Jakobsson PJ. Enzymes of the cyclooxygenase pathways of prostanoid biosynthesis. Chem Rev 2011;111:5821-5865.

23. Yamakawa K, Kamekura S, Kawamura N, Saegusa M, Kamei D, Murakami M et al. Association of microsomal prostaglandin E synthase 1 deficiency with impaired fracture healing, but not with bone loss or osteoarthritis, in mouse models of skeletal disorders. Arthritis Rheum 2008:58:172-183

24. Nakalekha C, Yokoyama C, Miura H, Alles N, Aoki K, Ohya $\mathrm{K}$ et al. Increased bone mass in adult prostacyclin-deficient mice. J Endocrinol 2010;204:125-133.

25. Traianedes K, Dallas MR, Garrett IR, Mundy GR, Bonewald LF. 5-Lipoxygenase metabolites inhibit bone formation in vitro. Endocrinology 1998:139:3178-3184.

26. Ren W, Dziak R. Effects of leukotrienes on osteoblastic cell proliferation. Calcif Tissue Int 1991;49:197-201.

27. Bonewald LF, Flynn M, Qiao M, Dallas MR, Mundy GR, Boyce BF. Mice lacking 5-lipoxygenase have increased cortical bone thickness. Adv Exp Med Biol 1997;433:299-302.

28. Garcia P, Schwenzer S, Slotta JE, Scheuer C, Tami AE, Holstein JH et al. Inhibition of angiotensin-converting enzyme stimulates fracture healing and periosteal callus formation role of a local renin-angiotensin system. Br J Pharmacol 2010;159:1672-1680.

29. Klein RF, Allard J, Avnur Z, Nikolcheva T, Rotstein D, Carlos AS et al. Regulation of bone mass in mice by the lipoxygenase gene Alox15. Science 2004;303:229-232.

30. Ichikawa S, Koller DL, Johnson ML, Lai D, Xuei X, Edenberg HJ et al. Human ALOX12, but not ALOX15, is associated with BMD in white men and women. $J$ Bone Miner Res 2006;21: 556-564.

31. Cheung CL, Chan V, Kung AW. A differential association of ALOX15 polymorphisms with bone mineral density in pre- and post-menopausal women. Hum Hered 2008;65:1-8.

32. Xu Z, Choudhary S, Okada Y, Voznesensky O, Alander C, Raisz L et al. Cyclooxygenase-2 gene disruption promotes proliferation of murine calvarial osteoblasts in vitro. Bone 2007;41:68-76.

33. Gerstenfeld LC, Thiede M, Seibert K, Mielke C, Phippard D, Svagr B et al. Differential inhibition of fracture healing by non-selective and cyclooxygenase-2 selective non-steroidal antiinflammatory drugs. J Orthop Res 2003;21:670-675.

34. Dekel S, Lenthall G, Francis MJ. Release of prostaglandins from bone and muscle after tibial fracture. An experimental study in rabbits. J Bone Joint Surg Br 1981;63-B:185-189.

35. Gerstenfeld LC, Al-Ghawas M, Alkhiary YM, Cullinane DM, Krall EA, Fitch JL et al. Selective and nonselective cyclooxygenase-2 inhibitors and experimental fracture-healing. Reversibility of effects after short-term treatment. J Bone Joint Surg Am 2007;89:114-125.

36. Kobayashi T, Narumiya S. Function of prostanoid receptors: studies on knockout mice. Prostaglandins Other Lipid Mediat 2002;68-69:557-573.

37. Tager AM, Luster AD. BLT1 and BLT2: the leukotriene B(4) receptors. Prostaglandins Leukot Essent Fatty Acids 2003;69:123-134.

38. Li M, Healy DR, LiY, Simmons HA, Crawford DT, Ke HZ et al. Osteopenia and impaired fracture healing in aged EP4 receptor knockout mice. Bone 2005;37:46-54.

39. Zhang M, Ho HC, Sheu TJ, Breyer MD, Flick LM, Jonason JH et al. EP1(-/-) mice have enhanced osteoblast differentiation and accelerated fracture repair. $J$ Bone Miner Res 2011:26:792-802.

40. Li M, Ke HZ, Qi H, Healy DR, Li Y, Crawford DT et al. A novel, non-prostanoid EP2 receptorselective prostaglandin E2 agonist stimulates local bone formation and enhances fracture healing. J Bone Miner Res 2003;18:2033-2042.

41. Paralkar VM, Borovecki F, Ke HZ, Cameron KO, Lefker B, Grasser WA et al. An EP2 receptorselective prostaglandin E2 agonist induces bone healing. Proc Natl Acad Sci USA 2003:100:6736-6740.

42. Namikawa T, Terai $\mathrm{H}$, Hoshino M, Kato M, Toyoda $\mathrm{H}$, Yano $\mathrm{K}$ et al. Enhancing effects of a prostaglandin EP4 receptor agonist on recombinant human bone morphogenetic protein-2 mediated spine fusion in a rabbit model. Spine (Phila Pa 1976) 2007;32:2294-2299.

43. Tanaka M, Sakai A, Uchida S, Tanaka S, Nagashima M, Katayama T et al. Prostaglandin E2 receptor (EP4) selective agonist (ONO-4819.CD) accelerates bone repair of femoral cortex after drill-hole injury associated with local upregulation of bone turnover in mature rats. Bone 2004:34:940-948

44. Jones TR, Labelle M, Belley M, Champion E, Charette L, Evans J et al. Pharmacology of montelukast sodium (singulair), a potent and selective leukotriene D4 receptor antagonist. Can J Physiol Pharmacol 1995;73:191-201.

45. Currie GP, McLaughlin K. The expanding role of leukotriene receptor antagonists in chronic asthma. Ann Allergy Asthma Immunol 200697731-741quiz 741-2, 793.

46. Wixted JJ, Fanning PJ, Gaur T, O'Connell SL, Silva J, Mason-Savas A et al. Enhanced fracture repair by leukotriene antagonism is characterized by increased chondrocyte proliferation and early bone formation: a novel role of the cysteinyl LT-1 receptor. J Cell Physiol 2009;221:31-39. 
47. Cakici H, Hapa O, Gideroglu K, Ozturan K, Guven M, Yuksel HY et al. The effects of leukotriene receptor antagonist montelukast on histological, radiological and densitometric parameters of fracture healing. Eklem Hastalik Cerrahisi 2011;22:43-47.

48. Ramires R, Caiaffa MF, Tursi A, Haeggstrom JZ, Macchia L. Novel inhibitory effect on 5lipoxygenase activity by the anti-asthma drug montelukast. Biochem Biophys Res Commun 2004:324:815-821.
49. Ueda K, Saito A, Nakano H, Aoshima M, Yokota M, Muraoka R et al. Cortical hyperostosis following long-term administration of prostaglandin $E_{1}$ in infants with cyanotic congenital heart disease. J Pediatr 1980;97:834-836.

50. Hasturk H, Kantarci A, Ohira T, Arita M, Ebrahimi N, Chiang N et al. RvE1 protects from local inflammation and osteoclast- mediated bone destruction in periodontitis. FASEB $J$ 2006;20:401-403. 